\title{
Prognostic significance of neutrophil-to- lymphocyte ratio in esophageal cancer: a meta-analysis
}

This article was published in the following Dove Press journal:

OncoTargets and Therapy

10 April 2015

Number of times this article has been viewed

\author{
Xun Yang' \\ Ying Huang ${ }^{2}$ \\ Ji-Feng Feng' \\ Jin-Shi Liu' \\ 'Department of Thoracic Surgery, \\ ${ }^{2}$ Department of Operating Theatre, \\ Zhejiang Cancer Hospital, Hangzhou, \\ People's Republic of China
}

Background: The neutrophil-to-lymphocyte ratio (NLR) is a useful predictive factor in several cancers. However, the prognostic value of NLR in patients with esophageal cancer (EC) is still controversial. Therefore, it was necessary for us to perform a meta-analysis to evaluate the prognostic value of NLR in patients with EC.

Methods: A systematic literature search was performed by using Web of Science, PubMed Central, and Medline to evaluate the prognostic value of NLR in patients with EC. The deadline of our primary search was July 10, 2014. This meta-analysis was conducted in accordance with PRISMA guidelines. Pooled hazard ratio (HR) with 95\% confidence interval (CI) was used to assess the association of NLR and overall survival (OS) and disease-free survival (DFS).

Results: Six studies involving 1,633 patients were included in our meta-analysis. Our pooled results demonstrated that high NLR was associated with poor OS (HR: 1.54, 95\% CI: 1.32-1.80, $I^{2}=25.3 \%, P=0.254$ ) and DFS (HR: $1.74,95 \% \mathrm{CI}: 1.25-2.43, I^{2}=63.9 \%, P=0.096$ ). Subgroup analysis between NLR and OS was performed in a further investigation. When the patients were segregated according to country, sample size, and pathological type, high NLR was also significantly correlated with OS.

Conclusion: High NLR is associated with poor prognosis in patients with EC. NLR may be a significant predictive biomarker in patients with EC.

Keywords: esophageal cancer, neutrophil-to-lymphocyte ratio, prognosis, meta-analysis, overall survival, disease-free survival

\section{Introduction}

Esophageal cancer (EC) is one of the most common cancers worldwide. ${ }^{1}$ Although there is a significant improvement in the treatment of patients with $\mathrm{EC}$, the prognosis is still poor due to late diagnosis, rapid progression, and high rate of recurrence. . $^{2,3}$ Therefore, it is important for us to identify better predictive factors, especially serum predictive biomarkers, for prognosis in patients with EC.

In recent years, systemic inflammatory response has been shown to correlate with prognosis in various cancers. ${ }^{4,5}$ Various inflammatory biomarkers, such as cytokine, C-reactive protein (CRP), and Glasgow prognostic score, have been investigated in a variety of cancers. ${ }^{6-9}$ Recent studies have demonstrated that neutrophil-to-lymphocyte ratio (NLR) is associated with prognosis in various cancers. ${ }^{10-12}$ However, due to the inconsistent results, the prognostic value of NLR in EC remains controversial. ${ }^{13-20}$ Therefore, it was necessary for us to perform a meta-analysis to evaluate the prognostic value of NLR in patients with EC.

In this study, we conducted a meta-analysis to evaluate the prognostic value of NLR for survival in patients with EC. To the best of our knowledge, this is the first
Correspondence: Ji-Feng Feng Department of Thoracic Surgery, Zhejiang Cancer Hospital, No 38 Guangji Road, Hangzhou 310022, People's Republic of China Tel +86 57I 88128052

Email jifzhejiang@gmail.com 
meta-analysis to investigate the prognostic role of NLR in patients with EC.

\section{Materials and methods Literature search}

A systematic literature search was performed by using Web of Science, PubMed, and Medline to evaluate the prognostic value of NLR in patients with EC. The search strategy was based on combinations of the following search terms: ("neutrophil-lymphocyte ratio" or "neutrophil-to-lymphocyte ratio" or "neutrophil lymphocyte ratio" or "NLR") and ("esophageal cancer" or "esophageal carcinoma" or "EC"). The deadline of our primary search was July 10, 2014. Only human research was included in our meta-analysis. In addition, the reference lists of identified studies were also checked for further relevant studies.

\section{Inclusion/exclusion criteria}

Inclusion criteria were as follows: 1) patients were diagnosed as having EC by pathology; 2) the NLR was measured before treatment; 3) the NLR was measured by serum-based methods; 4) the relationship between NLR and prognosis (overall survival [OS] and disease-free survival [DFS]) was evaluated; 5) full-text studies without language limits. The exclusion criteria were 1) review, letter, case report, or nonhuman research; 2) insufficient data to extract the hazard ratios (HRs) and the $95 \%$ confidence intervals (CIs).

\section{Data extraction}

Data from each study were extracted independently by two authors (Yang and Huang). If the results reported had possible overlap, only the most recent or the most complete study was included in this study. The following data were collected for each study: author, year, sample, country, treatment, pathological type, cutoff level, TNM stage, follow-up, and HRs and 95\% CIs for the correlation between NLR and prognosis.

\section{Statistical analysis}

Pooled HRs and 95\% CIs were used to analyze the relationship between NLR and prognosis (OS and DFS). The heterogeneity of combined HRs was initially evaluated by $\chi$-square test and expressed by inconsistency index $I^{2}$. A significant heterogeneity was defined as $P<0.10$ or $I^{2}>50 \%{ }^{21} \mathrm{~A}$ combined HR $>1$ indicated a worse OS, and it was considered statistically significant if the $95 \%$ CI for the HR did not overlap. Publication bias was assessed using Begg's funnel plot and Egger's linear regression test. All statistical analyses

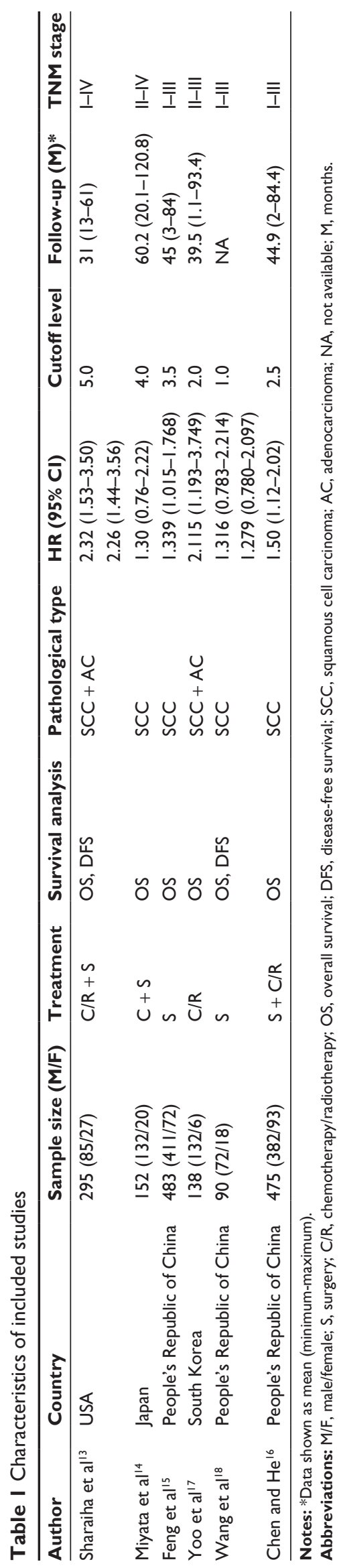




\begin{tabular}{|c|c|c|c|}
\hline Study & & HR $(95 \% \mathrm{Cl})$ & Weight (\%) \\
\hline Sharaiha et al ${ }^{13}$ & $\rightarrow$ & $2.32(1.53,3.50)$ & 14.40 \\
\hline Miyata et al ${ }^{14}$ & & $1.30(0.76,2.22)$ & 8.58 \\
\hline Feng et al ${ }^{15}$ & $\rightarrow$ & $1.34(1.01,1.77)$ & 32.02 \\
\hline Yoo et $\mathrm{al}^{17}$ & & $2.12(1.19,3.75)$ & 7.52 \\
\hline Wang et $\mathrm{al}^{18}$ & & $1.32(0.78,2.21)$ & 9.13 \\
\hline Chen and $\mathrm{He}^{16}$ & $\longrightarrow$ & $1.50(1.12,2.02)$ & 28.85 \\
\hline Overall $\left(I^{2}=25.3 \%, P=0.245\right)$ & 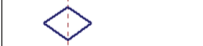 & $1.54(1.32,1.80)$ & 100.00 \\
\hline
\end{tabular}

Figure I Forest plot of the association between NLR and OS in EC.

Abbreviations: $\mathrm{HR}$, hazard ratio; $\mathrm{Cl}$, confidence interval; NLR, neutrophil-to-lymphocyte ratio; OS, overall survival; EC, esophageal cancer.

were conducted with Stata version 12.0 (Stata Corporation, College Station, TX, USA).

\section{Results}

According to the search strategies, a total of six eligible studies, including 1,633 patients with EC, were included in this meta-analysis. ${ }^{13-18}$ Sato et al ${ }^{19}$ demonstrated the correlation between NLR and the response to neoadjuvant chemotherapy. Thus, we excluded it from this study. Rashid et $\mathrm{al}^{20}$ reported the opposite conclusion. However, we could not extract the data from the manuscript because of insufficient data. Therefore, we excluded Rashid's study as well. The main characteristics of the six included studies are listed in Table 1.

All these six studies presented data on NLR and OS, ${ }^{13-18}$ but only two studies reported data on NLR and DFS. ${ }^{13,17}$ Our pooled results showed that high NLR was associated with poor OS (HR: $1.54,95 \%$ CI: $1.32-1.80, I^{2}=25.3 \%, P=0.245$ ) (Figure 1) and DFS (HR: 1.74, 95\% CI: $1.25-2.43, I^{2}=63.9 \%$, $P=0.096$ ) (Figure 2).

In a further investigation, subgroup analyses were performed (Figure 3). When stratified by "sample size," the " $>300$ " group yielded a HR of 1.41 , and the $95 \%$ CI was $1.15-1.73$ (Figure $3 \mathrm{~A}$ ). The " $\leq 300$ " group yielded a HR of 1.76 , and the $95 \%$ CI was 1.38-2.26 (Figure 3B). In the subgroup analysis by "country," we found that no matter the patients were Chinese (Figure 3C) or non-Chinese (Figure 3D), high NLR was still a poor predictor for OS. The subgroup analysis by "pathological type" showed that high NLR still yielded a worse OS (Figure 3E and F).

Sensitivity analysis showed that no single study could affect the pooled HRs in the present meta-analysis (Figure 4A). The Begg's funnel plot and Egger's linear regression test were performed to analyze the publication bias. There was no significant publication bias in our study (Begg's test: $P=0.707$, Egger's test: $P=0.521$ ) (Figure $4 \mathrm{~B}$ and $\mathrm{C}$ ).

\section{Discussion}

To the best of our knowledge, this is the first meta-analysis to investigate the prognostic role of NLR in patients with EC. In this meta-analysis, our pooled results demonstrated that high NLR was associated with poor OS (HR: $1.54,95 \% \mathrm{CI}$ : $1.32-1.80, I^{2}=25.3 \%, P=0.245$ ) and DFS (HR: $1.74,95 \% \mathrm{CI}$ : $\left.1.25-2.43, I^{2}=63.9 \%, P=0.096\right)$ in patients with EC. Subgroup analyses between NLR and OS were performed in a further investigation. When the patients were segregated according to country, sample size, and pathological type, high NLR was also significantly correlated with OS.

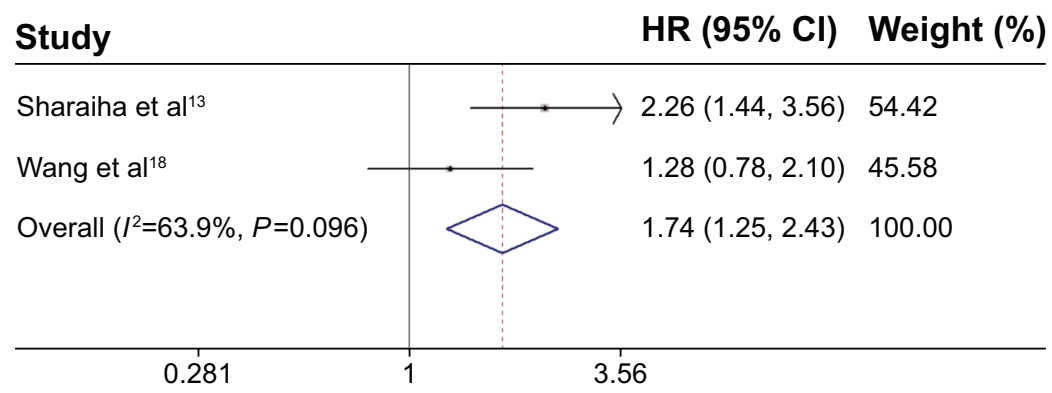

Figure 2 Forest plot of the association between NLR and DFS in EC.

Abbreviations: $\mathrm{HR}$, hazard ratio; $\mathrm{Cl}$, confidence interval; NLR, neutrophil-to-lymphocyte ratio; DFS, disease-free survival; EC, esophageal cancer. 

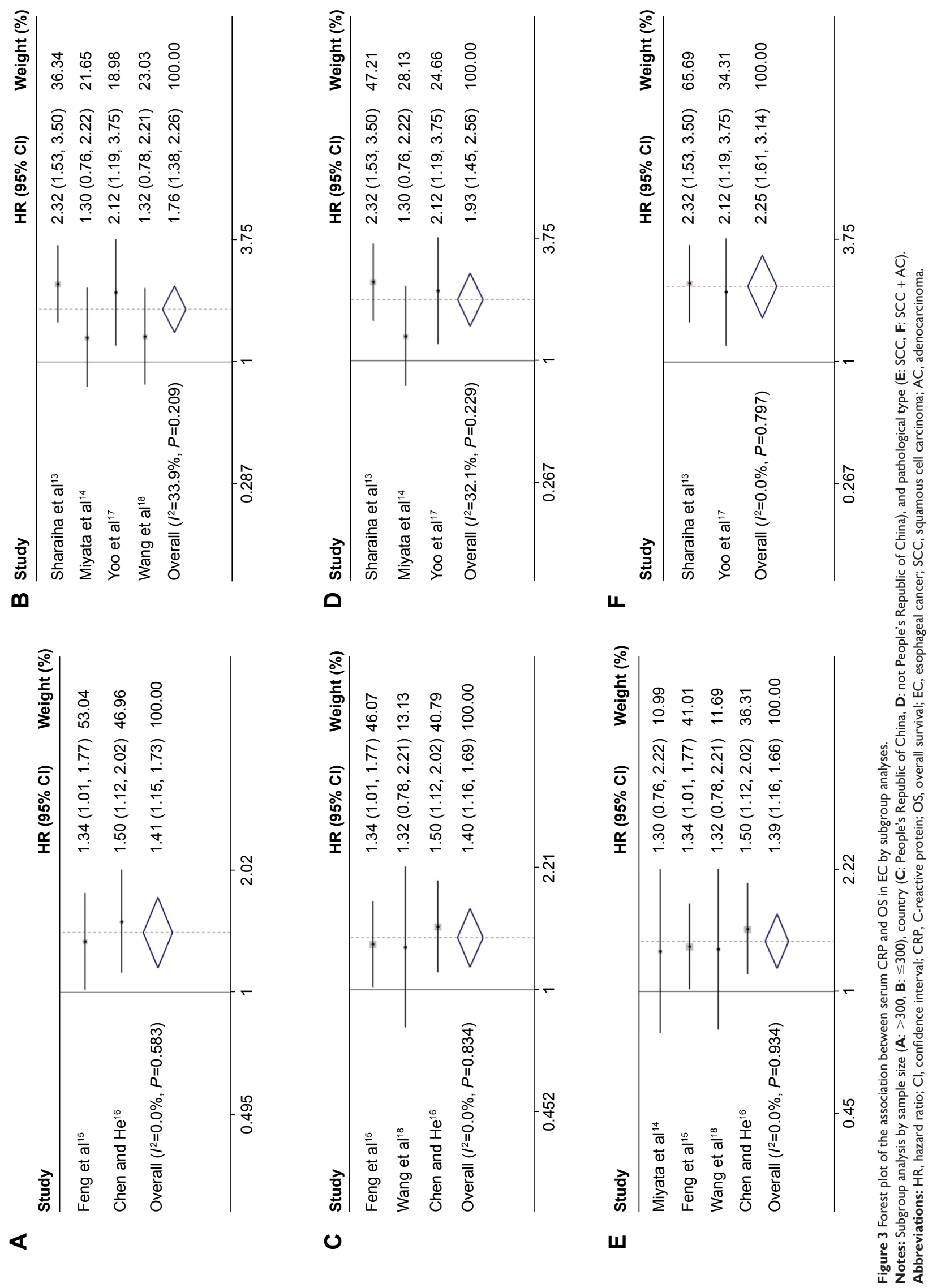
A

\section{Meta-analysis estimates, given named study is omitted}

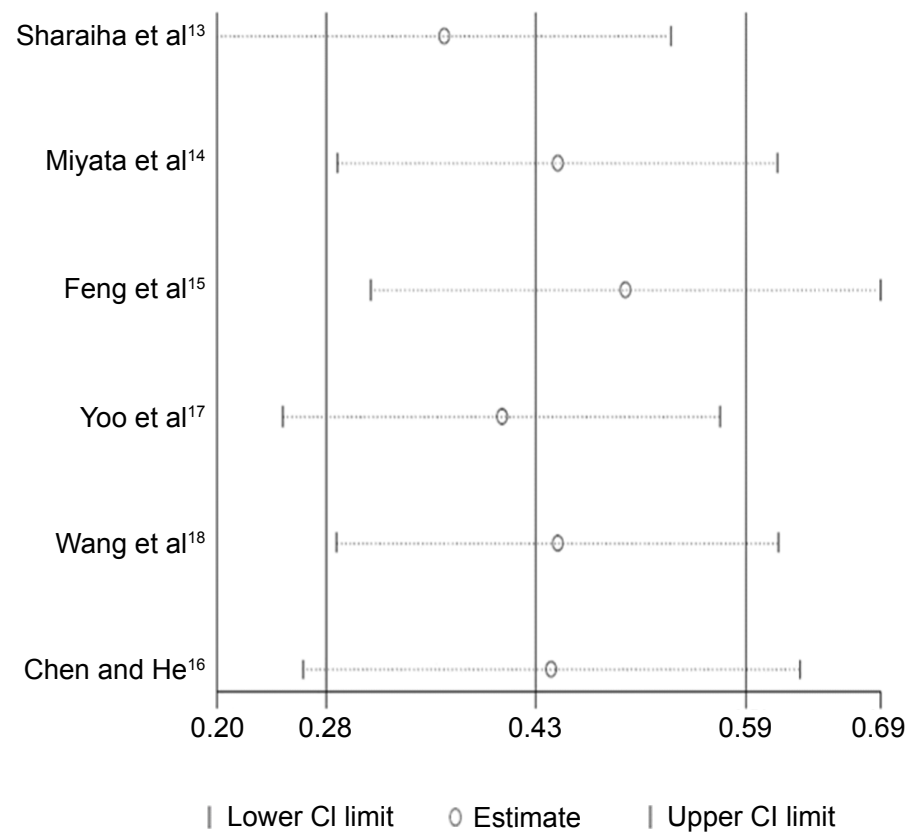

B
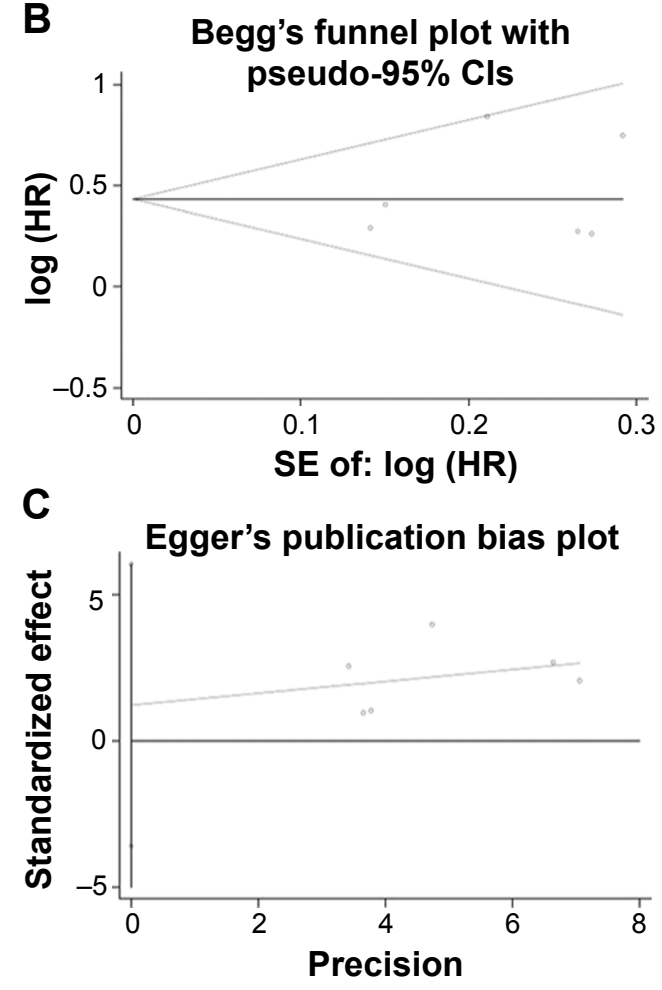

Figure 4 Sensitivity analysis (A) and funnel plot of publication biases (B and $\mathbf{C}$ ) on the relationship between NLR and OS in EC.

Abbreviations: $\mathrm{Cl}$, confidence interval; HR, hazard ratio; SE, standard error; NLR, neutrophil-to-lymphocyte ratio; OS, overall survival; EC, esophageal cancer.

Several meta-analyses demonstrated that NLR can be used as an independent predictive factor and can predict the prognosis of various cancers, including urinary cancer, ${ }^{22}$ hepatocellular carcinoma, ${ }^{23}$ and colorectal cancer. ${ }^{24}$ The results of our study are consistent with those of published reports. Furthermore, the sensitivity analysis showed that no single study could affect the pooled HRs in our metaanalysis. There was no significant publication bias in our study. This finding suggests that our meta-analysis was stable and reliable.

The mechanism of the prognostic value of NLR in patients with EC remains unclear. Several reports showed that NLR was associated with tumor-associated macrophages, which promote interleukin- $6 .{ }^{25}$ Furthermore, cancer has been shown to produce various cytokines, such as granulocyte colonystimulating factor, tumor necrosis factor- $\alpha$, transforming growth factor- $\beta$, interleukin- 1 , and interleukin- 6 , which may influence tumor-related neutrophilia. ${ }^{26,27}$

There were several limitations in our study. First, the number of included studies was limited. Second, the pooled HRs in our study between NLR and OS/DFS were not strong. Empirically, a HR $>2$ is considered a strongly predictive factor. Therefore, the results should be regarded with caution when using NLR to predict OS/DFS in EC. Moreover, because of the lack of a relevant prospective study, all of the included studies were retrospective. In addition, Rashid's study was important for our study. However, we excluded it due to insufficient data, which may have influenced our analysis. Therefore, more prospective studies are needed to confirm whether NLR is a prognostic factor in patients with EC.

In conclusion, our meta-analysis demonstrated that high NLR was significantly associated with poor OS and DFS in patients with EC. We conclude that NLR might serve as a useful biomarker for EC. However, further large prospective studies should be carried out to confirm whether NLR has a prognostic value in patients with EC.

\section{Disclosure}

The authors report no conflicts of interest in this work.

\section{References}

1. Ferlay J, Shin HR, Bray F, et al. Estimates of worldwide burden of cancer in 2008: GLOBOCAN 2008. Int J Cancer. 2010;127(12):2893-2917.

2. Feng JF, Huang Y, Zhao Q. Tumor length in elderly patients with esophageal squamous cell carcinoma: is it a prognostic factor? Ups J Med Sci. 2013;118(3):145-152.

3. Feng JF, Chen QX. Significance of the prognostic nutritional index in patients with esophageal squamous cell carcinoma. Ther Clin Risk Manag. 2014;10:1-7.

4. Mantovani A, Allavena P, Sica A, et al. Cancer-related inflammation. Nature. 2008;454(7203):436-444. 
5. Balkwill F, Mantovani A. Inflammation and cancer: back to Virchow? Lancet. 2001;357(9255):539-545.

6. Nakanishi H, Araki N, Kudawara I, et al. Clinical implications of serum C-reactive protein levels in malignant fibrous histiocytoma. Int J Cancer. 2002;99(2):167-170.

7. Platt JJ, Ramanathan ML, Crosbie RA, et al. C-reactive protein as a predictor of postoperative infective complications after curative resection in patients with colorectal cancer. Ann Surg Oncol. 2012;19(13): 4168-4177.

8. Vashist YK, Loos J, Dedow J, et al. Glasgow prognostic score is a predictor of perioperative and long-term outcome in patients with only surgically treated esophageal cancer. Ann Surg Oncol. 2011;18(4):1130-1138.

9. Feng JF, Zhao HG, Liu JS, et al. Significance of preoperative C-reactive protein as a parameter in patients with small cell carcinoma of the esophagus. Onco Targets Ther. 2013;6:1147-1151.

10. Walsh SR, Cook EJ, Goulder F, et al. Neutrophil-lymphocyte ratio as a prognostic factor in colorectal cancer. J Surg Oncol. 2005;91(3): 181-184.

11. Halazun KJ, Aldoori A, Malik HZ, et al. Elevated preoperative neutrophil to lymphocyte ratio predicts survival following hepatic resection for colorectal liver metastases. Eur J Surg Oncol. 2008;34(1):55-60.

12. Gomez D, Farid S, Malik HZ, et al. Pre-operative neutrophil-tolymphocyte ratio as a prognostic predictor after curative resection for hepatocellular carcinoma. World J Surg. 2008;32(8):1757-1762.

13. Sharaiha RZ, Halazun KJ, Mirza F, et al. Elevated preoperative neutrophil:lymphocyte ratio as a predictor of postoperative disease recurrence in esophageal cancer. Ann Surg Oncol. 2011;18(12): 3362-3369.

14. Miyata H, Yamasaki M, Kurokawa Y, et al. Prognostic value of an inflammation-based score in patients undergoing pre-operative chemotherapy followed by surgery for esophageal cancer. Exp Ther Med. 2011;2(5):879-885.

15. Feng JF, Huang Y, Chen QX. Preoperative platelet lymphocyte ratio (PLR) is superior to neutrophil lymphocyte ratio (NLR) as a predictive factor in patients with esophageal squamous cell carcinoma. World J Surg Oncol. 2014;12:58.

16. Chen H, He J. Preoperative neutrophil-to-lymphocyte ratio as a prognostic predictor after radical resection of esophageal squamous cell carcinoma. Zhonghua Zhong Liu Za Zhi. 2014;36(4):294-297. Chinese.
17. Yoo EJ, Park JC, Kim EH, et al. Prognostic value of neutrophilto-lymphocyte ratio in patients treated with concurrent chemoradiotherapy for locally advanced oesophageal cancer. Dig Liver Dis. 2014;46(9):846-853.

18. Wang J, Jia Y, Wang N, et al. The clinical significance of tumor infiltrating neutrophils and neutrophil-to-CD8+ lymphocyte ratio in patients with resectable esophageal squamous cell carcinoma. $J$ Transl Med. 2014;12:7.

19. Sato H, Tsubosa Y, Kawano T. Correlation between the pretherapeutic neutrophil to lymphocyte ratio and the pathologic response to neoadjuvant chemotherapy in patients with advanced esophageal cancer. World J Surg. 2012;36(3):617-622.

20. Rashid F, Waraich N, Bhatti I, et al. A pre-operative elevated neutrophil:lymphocyte ratio does not predict survival from oesophageal cancer resection. World J Surg Oncol. 2010;8:1.

21. Higgins JP, Thompson SG, Deeks JJ, et al. Measuring inconsistency in meta-analyses. BMJ. 2003;327(7414):557-560.

22. Wei Y, Jiang YZ, Qian WH. Prognostic role of NLR in urinary cancers: a meta-analysis. PLoS One. 2014;9:e92079.

23. Xiao WK, Chen D, Li SQ, et al. Prognostic significance of neutrophillymphocyte ratio in hepatocellular carcinoma: a meta-analysis. $B M C$ Cancer. 2014;14:117.

24. Li MX, Liu XM, Zhang XF, et al. Prognostic role of neutrophilto-lymphocyte ratio in colorectal cancer: a systematic review and metaanalysis. Int J Cancer. 2014;134(10):2403-2413.

25. Canna K, McArdle PA, McMillan DC, et al. The relationship between tumour T-lymphocyte infiltration, the systemic inflammatory response and survival in patients undergoing curative resection for colorectal cancer. Br J Cancer. 2005;92(4):651-654.

26. Kusumanto YH, Dam WA, Hospers GA, et al. Platelets and granulocytes, in particular the neutrophils, form important compartments for circulating vascular endothelial growth factor. Angiogenesis. 2003;6(4):283-287.

27. Klinger MH, Jelkmann W. Role of blood platelets in infection and inflammation. J Interferon Cytokine Res. 2002;22(9):913-922.
OncoTargets and Therapy

\section{Publish your work in this journal}

OncoTargets and Therapy is an international, peer-reviewed, open access journal focusing on the pathological basis of all cancers, potential targets for therapy and treatment protocols employed to improve the management of cancer patients. The journal also focuses on the impact of management programs and new therapeutic agents and protocols on

\section{Dovepress}

patient perspectives such as quality of life, adherence and satisfaction The manuscript management system is completely online and includes a very quick and fair peer-review system, which is all easy to use. Visit http://www.dovepress.com/testimonials.php to read real quotes from published authors. 